\title{
Prescriptions médicamenteuses usuelles en médecine et chirurgie orales : Etude rétrospective régionale sur l'état des lieux des prescriptions des chirurgiens-dentistes de la région Pays de la Loire.
}

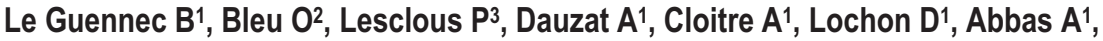 \\ Kimakhe $\mathrm{S}^{4}$ \\ 1. Service d'Odontologie Restauratrice et Chirurgicale, Unité Fonctionnelle de Chirurgie Orale, Faculté de Chirurgie Dentaire de \\ Nantes (France). (AHU) - CHU Nantes \\ 2. Faculté de Chirurgie Dentaire de Nantes (TCEO1) - CHU Nantes \\ 3. Service d'Odontologie Restauratrice et Chirurgicale, Unité Fonctionnelle de Chirurgie Orale, Faculté de Chirurgie Dentaire de \\ Nantes (France). (PU-PH) - CHU Nantes \\ 4. Service d'Odontologie Restauratrice et Chirurgicale, Unité Fonctionnelle de Chirurgie Orale, Faculté de Chirurgie Dentaire de \\ Nantes (France). (MCU-PH) - CHU Nantes
}

\section{Introduction}

Le praticien détient un droit de prescription limité à sa sphère de compétence et donc à sa pratique professionnelle. Pour chaque profession médicale, des recommandations sont éditées et régulièrement mises à jour selon les données actuelles de la science, permettant de guider le praticien dans ses prescriptions sans toutefois le priver de son libre choix. II existe peu ou pas de données concemant la mise en pratique de ces recommandations concernant les prescriptions médicamenteuses chez le en pratique bucco-dentaire.

\section{Matériel et méthodes}

Une étude épidémiologique observationnelle descriptive et rétrospective sur les prescriptions médicamenteuses des chirurgiens-dentistes de la région Pays de la Loire a été menée sur l'année 2013 à partir des données fournies par l'Assurance Maladie. Cette étude s'est limitée aux classes de médicaments pour lesquelles la profession dispose de recommandations de bonne pratique : les antibiotiques, les antalgiques et les anti-inflammatoires. Une analyse statistique descriptive a été réalisée

\section{Résultats}

Les antibiotiques sont les médicaments les plus prescrits : $55.5 \%$ des prescriptions étudiées et parmi ceux-ci, l'amoxicilline est la molécule la plus prescrite (32\%) au sein de 60 molécules ou associations de molécules différentes. Parmi les antalgiques, le paracétamol est la molécule la plus prescrite $32.6 \%$ devant libuprofène (20\%). Enfin, parmi les antiinflammatoires stéroïdiens, la prednisone et la prednisolone représentent plus de $60 \%$ des prescriptions.

\section{Discussion}

Malgré ses limites, cette étude a quand même permis de constater qu'une majorité des prescriptions, classe par classe, figure dans les recommandations de bonne pratique disponibles. Elle a aussi identifié la persistance de certaines « habitudes » de prescriptions ainsi que des prescriptions inadéquates. Enfin, cette étude a mis en lumière, une fois de plus, l'importance de la formation continue et la remise à jour des prescriptions médicamenteuses et plus particulièrement celles des antibiotiques.

\section{Conclusion}

De trop nombreux praticiens se réfèrent encore à leur seule expérience personnelle ou à des connaissances empiriques. Or, il est de la responsabilité des chirurgiens-dentistes, et des prescripteurs en général, de prendre conscience que le mésusage médicamenteux n'est pas uniquement lié à l'automédication mais qu'll est également le fruit de la méconnaissance des praticiens envers l'évolution des recommandations, au détriment du rapport bénéfices/risques, qu'il soit individuel ou collectif.

chirurgie.orale.leguennec@gmail.com

(C) The authors, published by EDP Sciences. This is an Open Access article distributed under the terms of the Creative Commons Attribution License 4.0 (http://creativecommons.org/licenses/by/4.0/) 